Check for updates

Cite this: RSC Adv., 2019, 9, 27

Received 22nd October 2018 Accepted 5th December 2018

DOI: $10.1039 / \mathrm{c} 8 \mathrm{ra} 08738 \mathrm{e}$

rsc.li/rsc-advances

\section{Facile approach for a robust graphene/silver nanowires aerogel with high-performance electromagnetic interference shielding $\dagger$}

\author{
Xiaoting Liu, ${ }^{a}$ Tianrui Chen, ${ }^{a}$ Hao Liang, ${ }^{a}$ Faxiang Qin, ${ }^{a}$ Hui Yang ${ }^{\text {ab }}$ \\ and Xingzhong Guo (D) *ab
}

Robust graphene/silver nanowires (AgNWs) hybrid aerogels were fabricated by facile processes including mixing directly, reducing, and ambient pressure drying. The mechanical properties and electromagnetic interference (EMI)-shielding performance of the resultant hybrid aerogels were investigated in detail. Because silver nanowires with a high aspect ratio have been acting as crosslinkers to bridge twodimensional graphene sheets, a highly porous and electrically conducting framework can resist high external loading to prevent major deformation and act as an express way for electron transport. Consequently, the hybrid aerogel exhibits large mechanical strength of $42 \mathrm{kPa}, 35$ times larger than that of the neat reduced graphene oxide aerogel $(1.2 \mathrm{kPa})$, which can resist great damage. More importantly, the as-prepared aerogel possesses high EMI-shielding performance of up to $\sim 45.2 \mathrm{~dB}$ due to its unique nanostructure and good electrical properties. These results indicate that graphene/AgNWs hybrid aerogel prepared using this simplified method promises to be an ideal functional component for mechanically robust and high-performance EMI-shielding nanocomposites.

\section{Introduction}

With extensive applications in electronic and wireless technologies, materials with high electromagnetic interference (EMI)shielding performance have been in high demand to prevent pollution by electromagnetic waves, affecting the performance and lifetime of electronic devices as well as human health. ${ }^{1-4}$ Conventionally, the strategy for EMI-shielding in practical applications involves metal-based materials equipped with prominent EMI-shielding effectiveness (SE) and a convenient assembly process. Yet high density and poor environmental stability have severely impeded their further development..$^{5-8}$ Apart from excellent EMI-SE, a light weight and chemical stability are other critical factors for electromagnetic shielding materials, especially in the automobile and portable electronics industries.

Due to the low density, superior electrical conductivity, and good chemical resistance, carbon materials (especially graphene and carbon nanotubes) have been designed to combat EMI interference for controlling or mitigating electromagnetic-

${ }^{a}$ State Key Laboratory of Silicon Materials, School of Materials Science and Engineering, Zhejiang University, Hangzhou 310027, PR China. E-mail: msewj01@ zju.edu.cn; Fax: +86-571-87953313; Tel: +86-571-87953313

${ }^{b}$ Zhejiang-California International NanoSystems Institute, Zhejiang University, Hangzhou 310058, PR China

$\dagger$ Electronic supplementary information (ESI) available. See DOI: 10.1039/c8ra08738e radiation pollution and have achieved substantial progress. ${ }^{9}$ Chemical vapour deposition (CVD) ${ }^{10-13}$ and high-temperature carbonization of carbon precursors are the main strategies to achieve three-dimensional porous materials with a defect-free structure for EMI-shielding applications. Wei et al. ${ }^{10}$ reported a CNT-multilayered graphene edge plane core-shell hybrid foam using CVD methods for high EMI-SE; the hybrids exhibited great prospects as nano-reinforcements for fabricating highstrength polymer-based composites. Shen et al. ${ }^{7}$ and Xi et al. ${ }^{14}$ used thermal annealing at 2000 and $3000{ }^{\circ} \mathrm{C}$ for graphene oxide films to obtain high shielding performance. Nevertheless, either CVD or carbonization consumes a large amount of energy in materials processing, hindering the large-scale application of EMI-shielding materials. Moreover, graphene aerogels reduced by highly toxic reagents such as hydrazine hydrate and hydroiodic acid have limited SE below $35 \mathrm{~dB}$ over the entire frequency range. ${ }^{15-19}$ Other relatively simple methods reported in the literature have no clear electromagnetic $\mathrm{SE}^{.23-30}$ Thus, it is urgent to develop a scalable and economical way to prepare impressive materials for highly efficient shielding applications.

In this work, we successfully fabricated robust reduced graphene oxide (RGO)/AgNWs hybrid aerogels using a facile and energy-saving method without a high-temperature process or polluted reagent. Based on the bridging effect of mechanically strong AgNWs with a high aspect ratio, the hybrid aerogel shows increased mechanical strength ( $42 \mathrm{kPa}$ ) by 35 times compared to the neat RGO aerogel (1.2 kPa). Moreover, the addition of AgNWs for higher carrier concentration endows the aerogels 
with good electrical properties. Therefore, the as-prepared RGO/ AgNWs hybrid aerogel with density as low as $0.019 \mathrm{~g} \mathrm{~cm}^{-3}$ exhibits remarkable EMI-SE, approximately $45.2 \mathrm{~dB}$ in the frequency range of $8.2-12.4 \mathrm{GHz}$, a remarkable increase of $264 \%$ compared with pure RGO aerogel (roughly $17.1 \mathrm{~dB}$ ).

\section{Experimental}

\subsection{Materials}

Graphene oxide (GO, $10 \mathrm{mg} \mathrm{mL}{ }^{-1}$ ) dispersion was obtained from Hangzhou Gaoxi Technology Co., Ltd. Silver nitrate $\left(\mathrm{AgNO}_{3}, 99 \%\right)$, ethylene glycol (EG, 99.8\%), polyvinylpyrrolidone (PVP, $M_{\mathrm{w}}$ of 360000 ), and ferric chloride $\left(\mathrm{FeCl}_{3}, 98 \%\right)$ were purchased from Aladdin Ind Co. in China. Ethanol and ascorbic acid were provided by Sinopharm Chemical Reagent Co., Ltd. All chemicals were used without further purification.

\subsection{Preparation of silver nanowires}

Silver nanowires were synthesized by reducing $\mathrm{AgNO}_{3}$ with EG in the presence of PVP and $\mathrm{FeCl}_{3}$. In the synthesis process, $22 \mathrm{~mL}$ of EG containing $0.08 \mathrm{~g}$ of PVP was added to a roundbottom flask and heated for $30 \mathrm{~min}$ at $140{ }^{\circ} \mathrm{C}$. Then, freshly prepared $2.5 \mathrm{~mL}$ of $600 \mu \mathrm{M} \mathrm{FeCl}_{3}$ in EG and $3 \mathrm{~mL}$ of $0.06 \mathrm{~g} \mathrm{~mL}^{-1}$ $\mathrm{AgNO}_{3}$ in EG were injected dropwise into the above solution within $1 \mathrm{~min}$. The temperature was maintained under vigorous stirring for $1 \mathrm{~h}$. After nanowire formation, the solution was cooled to room temperature. Then, $100 \mathrm{~mL}$ of acetone was poured into the solution to static settlement for at least $12 \mathrm{~h}$, after which the precipitate was obtained by removing the liquid supernatant. The precipitate was centrifuged at $2000 \mathrm{rpm}$ for 6 min using ethanol for several times to remove excess solvent and other impurities in the supernatant. Later, AgNWs were resuspended in water to obtain a certain concentration of AgNWs.

\subsection{Preparation of RGO/AgNWs hybrid aerogels}

A simplified and cost-effective fabrication process of hybrid aerogel was introduced in this paper. First, $2 \mathrm{~mL}$ of GO aqueous dispersion was poured into a glass vial; then, AgNWs with different masses were added by adjusting the concentration of the AgNWs dispersion to obtain $4 \mathrm{~mL}$ of mixed solution before being stirred continuously until a homogenous GO/AgNWs solution formed. The mass ratio of GO and AgNWs in the mixed solution was $8: 1,4: 1,2: 1,1: 1$, and $1: 2$, respectively. As a reducing agent, $40 \mathrm{mg}$ of ascorbic acid was dissolved into the mixed solution later. Second, a sealed glass vial was heated for $1 \mathrm{~h}$ at $70{ }^{\circ} \mathrm{C}$ to synthesize partially reduced graphene hydrogel. Then, the weak hydrogels were held at $-20{ }^{\circ} \mathrm{C}$ for $8 \mathrm{~h}$ to ensure complete freezing. Third, the thawed hydrogels were further reduced for $5 \mathrm{~h}$ at $95^{\circ} \mathrm{C}$ to obtain fully reduced graphene hydrogels. Finally, the hydrogels were washed in water and dried in an oven at $60{ }^{\circ} \mathrm{C}$ for $24 \mathrm{~h}$ to obtain ambient-pressuredried aerogels. Pure RGO aerogel was prepared for comparison.

\subsection{Characterization}

Sample morphologies were characterized by scanning electron microscopy (SEM; SU-8010, Hitachi, Japan) and transmission electron microscopy (TEM; HT-7700, Hitachi, Japan). Raman microscopy (LabRAM HR Evolution, France) was performed at $532 \mathrm{~nm}$ laser excitation. X-ray photoelectron spectroscopy (XPS; 250XI, Japan) was used to identify the elemental compositions of RGO and RGO/AgNWs aerogels. X-ray diffractometry (XRD; APEXII, Bruker, Germany) was used to identify the crystal structure of obtained samples using Ni-filtered $\mathrm{Cu} \mathrm{K} \alpha$ radiation $(k=0.154 \mathrm{~nm})$. The mechanical properties of aerogels were tested using a dynamic mechanical analyzer (DMA; Q800, USA) at a strain ramp of $5 \%$ per min. The electrical conductivity was calculated from resistance, which was measured using a universal meter. EMI-shielding performance was measured in the frequency ranges of $8.2-12.4 \mathrm{GHz}$ and $12.4-18 \mathrm{GHz}$ at room temperature using a vector network analyzer (VNA; R\&s ZNB20) combined with two waveguide-to-coaxial adaptors connected face to face. The VNA was properly calibrated before measuring the scattering parameters $S$. Samples were cut into $22.9 \times 10.2$ $\times 5.0 \mathrm{~mm}$ and $15.8 \times 7.9 \times 5.0 \mathrm{~mm}$ (length $\times$ width $\times$ thickness) pieces to fit the waveguide holders corresponding to the two frequency band mentioned above, respectively. EMI shielding performance including total $\mathrm{SE}$, SE reflection, and $\mathrm{SE}$ absorption (denoted, respectively, as $\mathrm{SE}_{\mathrm{T}}, \mathrm{SE}_{\mathrm{R}}$, and $\mathrm{SE}_{\mathrm{A}}$ ) was calculated based on scattering parameters $\left(S_{11}\right.$ and $\left.S_{21}\right)$.

\section{Results and discussion}

\subsection{Morphologies and characteristics of RGO/AgNWs hybrid aerogels}

Fig. 1a illustrates the fabrication process of RGO/AgNWs aerogel and corresponding digital photos. The GO sheets and AgNWs were dispersed stably in aqueous solution individually. By strong agitation, a homogenous GO/AgNWs mixed aqueous solution was obtained. During the pre-reduction procedure, the complex solution was converted to a weak, partially cross-linked hydrogel due to slight restacking of RGO sheets. Further reduction followed, the removal of more oxygen-containing groups on the GO sheets promoted an increase in hydrophobic and $\pi-\pi$ interactions, ${ }^{31,32}$ and RGO sheets agglomerated

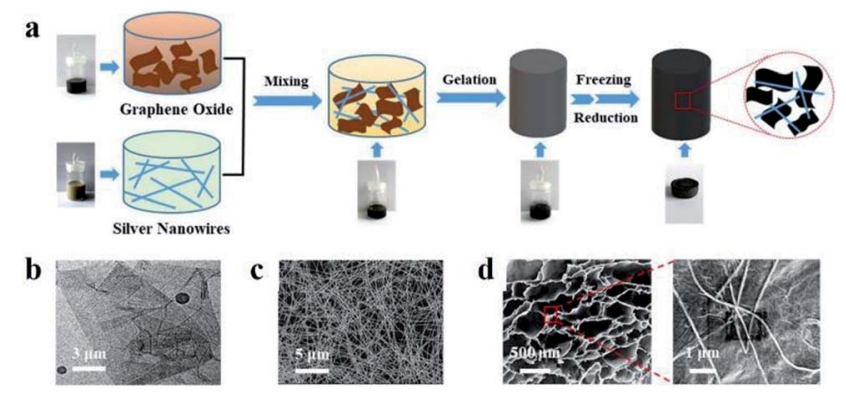

Fig. 1 (a) Schematic illustration of fabrication process of AgNWs/GO hybrid aerogel and corresponding digital photos; (b), (c) typical SEM and TEM images of GO and AgNWs individually; (d) SEM images of composite aerogel at different magnifications. 
and generated a stable monolithic assembly that floated completely in water. Throughout this process, the color of the sample in the vial changed from brown to black at a macroscopic level. Typical TEM and SEM images are displayed in Fig. $1 \mathrm{~b}$ and $\mathrm{c}$ and $\mathrm{S} 1 \uparrow$ to characterize the morphologies of the GO sheets, AgNWs, and GO/AgNWs in the aqueous solution. The sizes of GO sheets were $5-8 \mu \mathrm{m}$ with folded wrinkles dispersed over the substrate. AgNWs with an approximate diameter of $60 \mathrm{~nm}$ was obtained with a length ranging from 20 to $30 \mu \mathrm{m}$, playing a critical role as a bridge in the linkage of GO sheets. In the mixed solution, nanowires intersected with each other and attached firmly to the wrinkled GO layers. As shown in Fig. 1d, the obtained 3D network-structured RGO/AgNWs hybrid aerogel exhibited an abundant porous structure, with the pore size distributed over a range of a few microns to several hundred microns. With further magnification, several nanowires were observed on the GO lamellas consisting of pore walls and were interwoven together, indicating that AgNWs and RGO combined well and the RGO/AgNWs hybrid aerogels were prepared successfully.

The microstructure and chemical compositions of the asprepared aerogels were revealed in detail by XPS, Raman spectroscopy, and XRD. In XPS spectra (Fig. 2a), GO and RGO aerogels showed a noticeable carbon peak at $\sim 284 \mathrm{eV}$ and oxygen peaks at $\sim 532 \mathrm{eV}$. However, the $\mathrm{C} / \mathrm{O}$ atom ratio increased remarkably from 2.6 of GO to 6.6 after reduction, suggesting virtually successful elimination of oxygen functional groups. The high-resolution $C$ 1s scan spectra (inset in Fig. 2a) was further fitted into three subpeaks at $\sim 284.6, \sim 286.5 \mathrm{eV}$, and $\sim 288.3 \mathrm{eV}$, belonging to $\mathrm{C}-\mathrm{C} / \mathrm{C}=\mathrm{C}, \mathrm{C}-\mathrm{O}-\mathrm{C} / \mathrm{C}-\mathrm{OH}$, and $\mathrm{C}=\mathrm{O} / \mathrm{O}-$ $\mathrm{C}=\mathrm{O}$, respectively. High-intensity peaks of $\mathrm{C}-\mathrm{O}-\mathrm{C} / \mathrm{C}-\mathrm{OH}$ and $\mathrm{C}=\mathrm{O} / \mathrm{O}-\mathrm{C}=\mathrm{O}$ were observed in the $\mathrm{GO}$ aerogel; only a $\mathrm{C}-\mathrm{C} / \mathrm{C}=$ $\mathrm{C}$ component appeared in the RGO aerogel, verifying efficient deoxygenation. ${ }^{33}$ Moreover, the two peaks in the $\mathrm{Ag} 3 \mathrm{~d}$ XPS spectrum of hybrid aerogels at $368.1 \mathrm{eV}$ and $374.1 \mathrm{eV}$ (Fig. 2b)
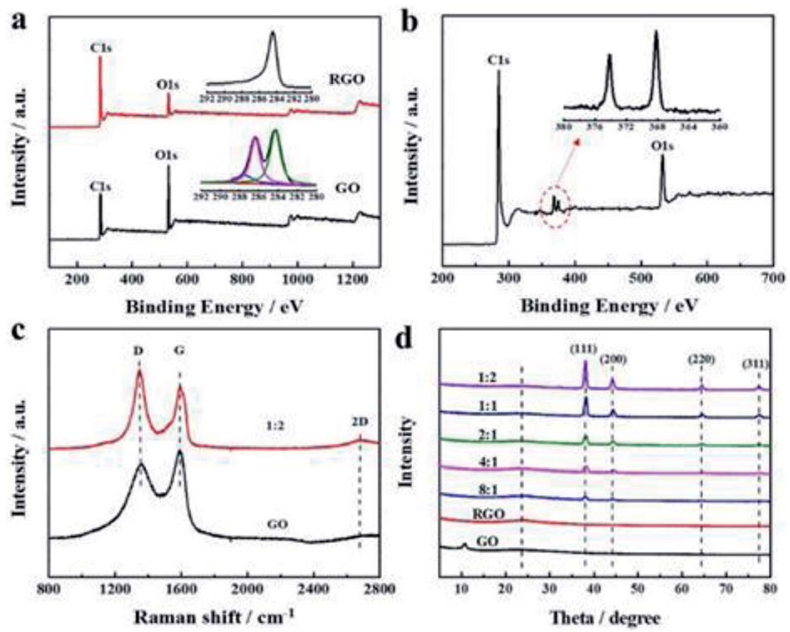

Fig. 2 XPS analysis of (a) GO, RGO aerogel and (b) hybrid aerogel of $1: 2$ (insets: high-resolution $\mathrm{C} 1 \mathrm{~s}$ and $\mathrm{Ag} 3 \mathrm{~d}$ spectrum); (c) Raman spectra of GO and hybrid aerogel of 1 : 2; (d) XRD patterns of GO and all hybrid aerogels. corresponded to $\mathrm{Ag} 3 \mathrm{~d}_{5 / 2}$ and $\mathrm{Ag} 3 \mathrm{~d}_{3 / 2}$, respectively, confirming the effective combination of AgNWs and RGO. Raman spectra of GO and the hybrid aerogel are displayed in Fig. 2c. The typical D band at $\sim 1350 \mathrm{~cm}^{-1}$ was linked to the presence of $\mathrm{sp}^{3}$ orbitals or edge structures, and the $\mathrm{G}$ band at $\sim 1590 \mathrm{~cm}^{-1}$ was associated with the $\mathrm{sp}^{2}$ graphitic lattice. ${ }^{34}$ The intensity ratio of the $\mathrm{D}$ band to the $\mathrm{G}$ band increased from 2.08 to 1.59 after reduction, indicating that the defects of graphene created by oxygencontaining groups were partially repaired in the as-prepared aerogel. Additionally, a broad and weak 2D band at $\sim 2700 \mathrm{~cm}^{-1}$ was observed in the Raman spectrum, related to the ordered carbon structures. The absence of a diffraction peak of GO at $10.9^{\circ}$ and the appearance of a diffraction peak of RGO at $24.5^{\circ}$ in XRD data (Fig. $2 \mathrm{~d}$ ) also evidenced the reduction in GO, consistent with the XPS and Raman results. In addition, peaks at $38.1^{\circ}, 45.6^{\circ}, 64.3^{\circ}$, and $77.6^{\circ}$ could be assigned to (111), (200), (220), and (311) reflections of a face-centered cubic phase, confirming the crystalline nature of AgNWs. ${ }^{2}$

\subsection{Mechanical performance of hybrid aerogels}

In the experimental process, we found that the addition of AgNWs provided strong resistance for deformation under external force. Mechanical measurements were performed to investigate the effect of AgNWs on the compressive performance of the hybrid aerogel. Fig. $3 a$ and $b$ show a comparison of the compressive properties of RGO/AgNWs hybrid aerogels with different mass ratios. As expected, all aerogels were compressed by $60 \%$, indicating good reversible compressibility. Notably, clear mechanical reinforcement was achieved with an increased loading content of silver nanowires; even when the mass ratio of GO and AgNWs was $1: 2$, a 35-fold improvement in compressive strength $(42 \mathrm{kPa})$ was obtained compared to that of the neat RGO aerogel $(1.2 \mathrm{kPa})$. Therefore, one-dimensional silver
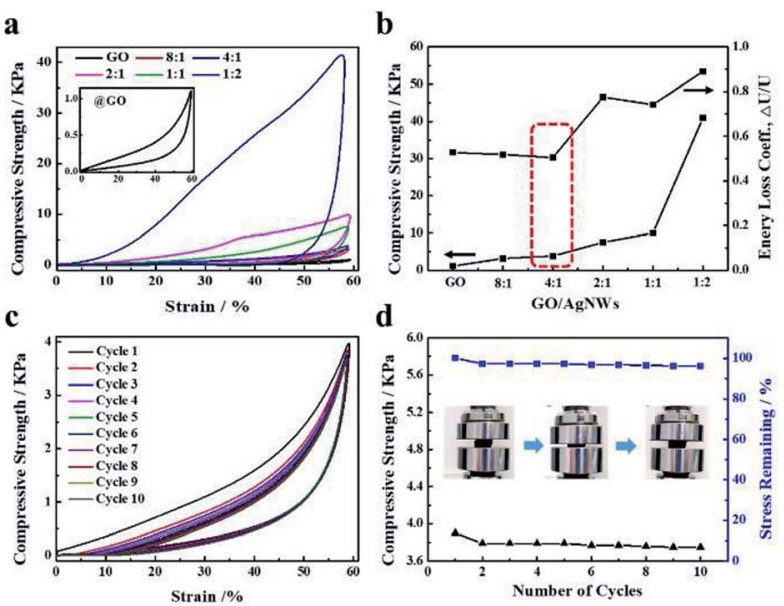

Fig. 3 Compressive behaviors of RGO/AgNWs hybrid aerogels: (a) typical stress-strain curves of different mass ratios of AgNWs and GO in composite aerogels; (b) compression strengths and energy loss coefficients of hybrid aerogels; (c) stress-strain curves during loading-unloading cycles at $60 \%$ strain by aerogel with GO/AgNWs mass ratio of $4: 1$; (d) compression strength and stress remaining ratio for each compression cycle. 
nanowires attached tightly to the surface of RGO sheets may act as a bridge to link with sheets, consolidating resistance deformation. Similarly, the trend of specific strength (the ratio of compressive strength to density) of RGO/AgNWs aerogels is shown in Fig. S2, $\uparrow$ which further indicates the dominate role of AgNWs on the strength of aerogel rather than the density. Furthermore, the energy loss coefficient, $\Delta U / U,{ }^{31}$ was calculated according to Fig. 3a. As displayed in Fig. 3b, the hybrid aerogel of $4: 1$ showed the lowest value in $\Delta U / U$, whereas the energy loss coefficient of the hybrid aerogel of $1: 2$ approached 0.9 , at which point dramatic energy depletion occurred. An effective interpenetrating network developed by combining onedimensional silver nanowires and 2D RGO sheets to provide good flexibility for recovery of the hybrid aerogel when the GO/ AgNWs mass ratio was $4: 1$. With increasing AgNWs loading content, great friction and adhesion between nanowires and sheets were produced during the loading-unloading process, leading to more energy loss. Corresponding to the lowest loss, the hybrid aerogel of $4: 1$ possessed optimal elasticity, as in the compressive stress-strain curve exhibited in Fig. 3c. After 10 compression cycles, the hybrid aerogel completely recovered its original shape with no obvious mechanical failure and maintained excellent recoverability as well as nearly constant strength (Fig. 3d).

\subsection{EMI-shielding performance and mechanism of hybrid aerogels}

Usually, main mechanisms for EMI-shielding are attributed to reflection, absorption attenuation, and multiple reflection of electromagnetic waves. ${ }^{\mathbf{1 1}}$ Interactions between electromagnetic waves and free charges on the surfaces of materials result in reflection of electromagnetic radiations, facilitating EMI-SE by reflection, $\mathrm{SE}_{\mathrm{R}}$. Absorption reveals the ability of materials to attenuate electromagnetic energy into thermal or internal energy, expressed by $\mathrm{SE}_{\mathrm{A}} \cdot{ }^{34}$ When the total $\mathrm{SE}$ exceeds $15 \mathrm{~dB}$, most multiply reflected waves can be further absorbed..$^{35}$ Thus, the reflection and absorption were taken as primary mechanisms for EMI-shielding in this work.

According to the literature, the total EMI-SE $\left(\mathrm{SE}_{\mathrm{T}}\right)$, is the logarithm of the power ratio of incident EM waves $\left(P_{\mathrm{I}}\right)$ to transmitted waves $\left(P_{\mathrm{T}}\right)$, measured by the $S$ parameters, $S_{11}$ and $S_{21}:^{36,37}$

$$
\begin{array}{r}
\mathrm{SE}_{\mathrm{T}}=10 \log \left(P_{\mathrm{I}} / P_{\mathrm{T}}\right) \\
\left|S_{11}\right|=\sqrt{\left(P_{\mathrm{R}} / P_{\mathrm{I}}\right)} \\
\left|S_{21}\right|=\sqrt{\left(P_{\mathrm{T}} / P_{\mathrm{I}}\right)}
\end{array}
$$

Here, $P_{\mathrm{R}}$ refers to the power of the reflected wave. The reflection coefficient $(R)$, transmission coefficient $(T)$, absorption coefficient $(A)$, and effective absorbance $\left(A_{\text {eff }}\right)$ can be calculated using eqn (4)-(7): ${ }^{38,39}$

$$
\begin{aligned}
& R=\left|S_{11}\right|^{\hat{2}} \\
& T=\left|S_{21}\right|^{\hat{2}}
\end{aligned}
$$

$$
\begin{gathered}
A=1-R-T \\
A_{\text {eff }}=(1-R-T) /(1-R)
\end{gathered}
$$

$\mathrm{SE}_{\mathrm{R}}$ and $\mathrm{SE}_{\mathrm{A}}$ can be derived from the power coefficients as follows: ${ }^{40-42}$

$$
\begin{gathered}
\mathrm{SE}_{\mathrm{R}}=-10 \log (1-R) \\
\mathrm{SE}_{\mathrm{A}}=-10 \log [T /(1-R)] \\
\mathrm{SE}_{\mathrm{T}}=\mathrm{SE}_{\mathrm{R}}+\mathrm{SE}_{\mathrm{A}}
\end{gathered}
$$

We tested the $S_{11}$ and $S_{21}$ parameters of as-prepared RGO and RGO/AgNWs hybrid aerogels to examine their EMIshielding performance at room temperature. Fig. 4 displays the EMI-shielding performance of hybrid aerogels with different mass ratios of $\mathrm{GO} / \mathrm{AgNWs}$ in the frequency range of 8.2-12.4 $\mathrm{GHz}$ ( $\mathrm{X}$ band). In general, an EMI-SE of $\sim 20 \mathrm{~dB}$ is needed for commercial applications. ${ }^{40}$ All hybrid aerogels demonstrated an $\mathrm{SE}_{\mathrm{T}}$ value larger than $20 \mathrm{~dB}$, which illustrates promise in practice. The average SE values appear in Fig. 4d. The pure RGO aerogel had a lower EMI-SE value of $17.1 \mathrm{~dB}$ because of its lower $\mathrm{SE}_{\mathrm{R}}$ and $\mathrm{SE}_{\mathrm{A}}$ values. As the content of AgNWs increased, the $\mathrm{SE}_{\mathrm{R}}$ value remained significantly low, and $\mathrm{SE}_{\mathrm{A}}$ values demonstrated sustained growth. When the mass ratio of GO/AgNWs was $1: 2$, the average EMI-SE reached $45.2 \mathrm{~dB}$, a tremendous improvement of $264 \%$. The as-prepared aerogel was therefore able to shield $99.997 \%$ of the incident electromagnetic waves. The improved EMI-SE could be ascribed to enhanced electrical conductivity (Fig. S3†), which was strongly associated with EMIshielding performance. More AgNWs loading could form an effective electron conductive network and led to a stronger interaction with incident electromagnetic waves, achieving an improved $\mathrm{SE}_{\mathrm{T}}$. To realize the stability of the resultant graphene/ AgNWs aerogel, we have tested the EMI SE of the hybrid aerogel
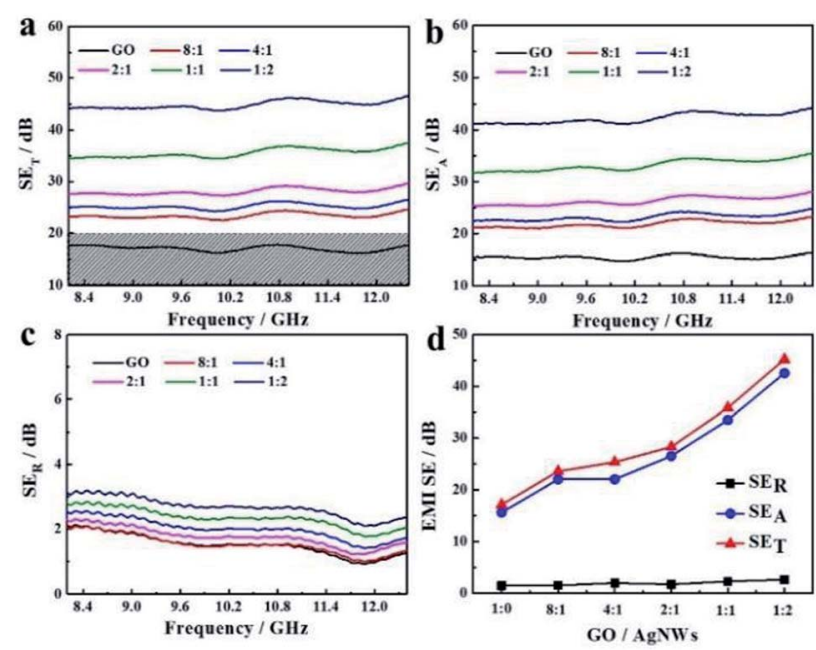

Fig. 4 EMI-shielding performances of RGO/AgNWs aerogel with different mass ratios in the frequency range from 8.2 to $12.4 \mathrm{GHz}$ : (a) $\mathrm{SE}_{\mathrm{T}}$, (b) $\mathrm{SE}_{\mathrm{A}}$, (c) $\mathrm{SE}_{\mathrm{R}}$; (d) average EMI-SE values from (a), (b), (c). 
$(1: 2)$ repeatedly after six months. The as-prepared aerogel owns excellent stability and its shielding performances (Fig. S4†) has no obvious change after six months. We guess the AgNWs are wrapped by the large GO nanosheets instead of being exposed to the air directly, protecting the AgNWs from oxidization. For lightweight and cellular shielding materials, the ratio of EMI-SE to density (so-called 'specific SE', SSE) ${ }^{\mathbf{1 1}}$ is usually introduced for performance comparison. With low densities ranging from 0.0059 to $0.019 \mathrm{~g} \mathrm{~cm}^{-3}$, the hybrid aerogels delivered high SSEs determined by normalizing $\mathrm{SE}_{\mathrm{T}}$ by the composite density (Fig. 5a). An extraordinarily high SSE of 3401 $\mathrm{dB} \mathrm{cm} \mathrm{cm}^{3} \mathrm{~g}^{-1}$ was achieved by the hybrid aerogel with GO/AgNWs mass ratio of $4: 1$, owing to its high $\mathrm{SE}_{\mathrm{T}}$ and low density. With increasing density, the SSEs suffered and exhibited a lower SSE of $2372 \mathrm{~dB} \mathrm{~cm} \mathrm{~cm}^{3} \mathrm{~g}^{-1}$ for the hybrid aerogel with a GO/AgNWs mass ratio of $1: 2$. Presumably, when the mass ratio of $\mathrm{GO} /$ AgNWs reached $4: 1$, one-dimensional silver nanowires served as a bridge to construct conductive networks with 2D RGO sheets based on electron hopping, effectively attenuating electromagnetic waves with microwave absorption while maintaining a lower structural density. However, continuing to add silver nanowires led to a rapid increase in density and relatively slow growth of EMI-SE values, resulting in lower SSE values. This demonstrates the existence of an optimal loading of constitutive fillers if both structural and shielding performance requires to be balanced. This finding is encouraging compared with other porous composites (Fig. 5b), which are fabricated by relatively eco-friendly methods, and more materials from available literatures with detailed information are listed in Table S1. $\dagger$ And the hybrid aerogels also displayed high EMIshielding performance and similar regularity in the range of 12.4-18 GHz (Ku band), shown in Fig. S5. $\dagger$ In combination with the results in Fig. 3, the hybrid aerogel presented good EMI-SE
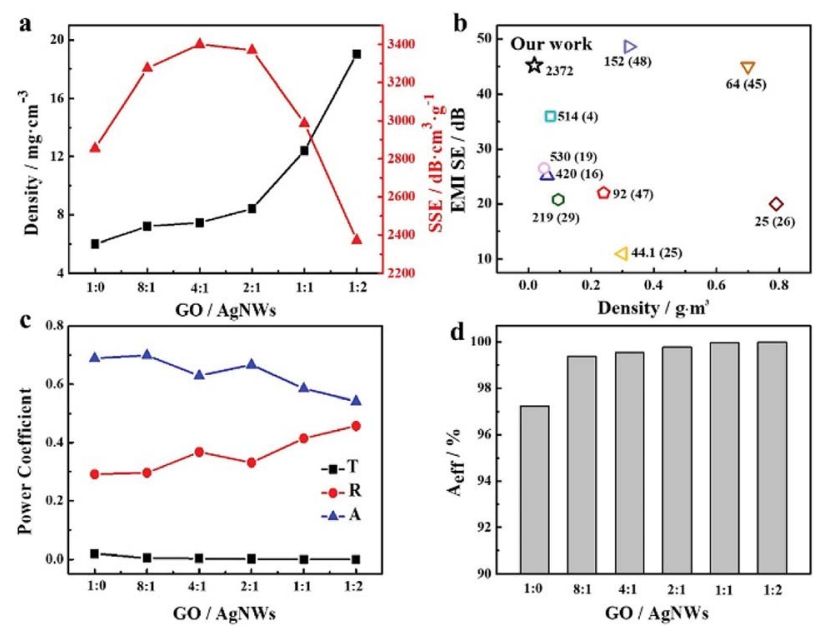

Fig. 5 (a) Density and SSE values of different hybrid aerogels; (b) Ashby chart plotting EMI-shielding performance versus density for typical porous composites (SSE values marked beside corresponding dots in chart; numbers in parenthesis represent relevant references). (c) Average $R, A, T$ values and $A_{\text {eff }}$ values (d) of hybrid aerogels with different mass ratios of GO/AgNWs in frequency range of 8.2-12.4 $\mathrm{GHz}$. and mechanical performances, further broadening the application of the as-prepared aerogels.

As revealed in Fig. 4, the absorption of electromagnetic waves exerted substantial influence on high EMI-shielding performance, which was strongly associated with the synergetic effect of electrical conductivity and multiple reflections in the aerogel. Generally, the shielding thickness $(d)$ and skin depth $(\delta)$ are used to evaluate $\mathrm{SE}_{\mathrm{A}}$ according to the formula $\mathrm{SE}_{\mathrm{A}}$ $=20(d / \delta) \log e=8.686(d / \delta)$, where $\delta$ is skin depth and defined as the electromagnetic energy reducing to $1 / e$ of the incident wave, described as $\delta=(\pi f \sigma \mu)^{-1 / 2}$ if $\sigma \gg 2 \pi f \varepsilon_{0}, \sigma$ is the electrical conductivity, $\varepsilon_{0}$ is the vacuum permittivity, and permeability $\mu$ $=\mu_{0} \mu_{\mathrm{r}}\left(\mu_{0}=4 \pi \times 10^{-7} \mathrm{H} \mathrm{m}^{-1}\right)$ and $\left(\mu_{\mathrm{r}}=1\right) .{ }^{43}$ As all samples were fabricated with uniform thickness, the $\mathrm{SE}_{\mathrm{A}}$ value was determined to be $\sigma$ in this work, which is linked to dielectric loss. A much higher $\mathrm{SE}_{\mathrm{A}}$ than $\mathrm{SE}_{\mathrm{R}}$ value suggests that absorption-dominant EMI-shielding occurred. For one, the current nanocomposite structure composed by RGO and AgNWs does not provide very high conductivity which can result in very exceptional reflection. For another, with RGO as substrate with suitable conductivity, the one dimensional nanowires help enhance the consumption of electromagnetic energy based on the dipolar and interfacial polarization. ${ }^{43}$ In addition, the existence of structural defects in hybrid aerogels can also serve as active sites for further improving the dielectric loss to promoting high absorption attenuation. ${ }^{\mathbf{1 0}}$

To better understand the EMI-shielding mechanism behind RGO/AgNWs hybrid aerogels, the corresponding power coefficients of reflectivity $(R)$, absorptivity $(A)$, transmissivity $(T)$, and effective absorption of electromagnetic waves ( $\left.A_{\text {eff }}\right)$ were calculated by $S$ parameters. ${ }^{44} R, A, T$ mirror the materials' capability to reflect, absorb and transmit electromagnetic waves, respectively. As displayed in Fig. $5 \mathrm{c}$, the $A$ and $T$ values decreased, and $R$ continued growing as the content of silver nanowires increased. Moreover, the value of $A$ was always higher than $R$, and the values of $A_{\text {eff }}$ (Fig. 5d) reached more than 99.5\%, aligning with the results in Fig. 4 where absorption was the primary shielding mechanism of RGO/AgNWs hybrid aerogels. However, from the variation trend in $R$ values, as reflection occurred first when the electromagnetic waves invaded, the reflection ability of RGO/AgNWs hybrid aerogels was enhanced with the construction of 3D networks between silver nanowires as well as between silver nanowires and RGO, despite a lower and nearly unchanged $\mathrm{SE}_{\mathrm{R}}$.

From a microstructure perspective, when entering the shielding materials, the free carriers (electrons and holes) interacted with incident waves and produced induced currents to dissipate energy from the electromagnetic waves. At the same time, the movement of induced currents generated an induced electromagnetic field in the materials to further decay incident waves. With growing AgNWs content, enhanced conductivity indicated more free carriers to move and consume electromagnetic waves. Certainly, AgNWs bridging between GO sheets provided efficient channels for electron transfer to accelerate the consumption of electromagnetic energy. High $\mathrm{SE}_{\mathrm{A}}$ benefitted from the attenuation of electromagnetic waves caused by induced currents and induced electromagnetic fields, 
which interacted with incident waves and then transformed into heat energy. In addition, the possibly shaped interconnected 3D network forms intricate structure and hence can redistribute the electromagnetic field, which further promotes the dissipation of electromagnetic energy. It follows these analyses that a proper absorbing structure design can capitalize on both the materials property and structure advantage. The material-structure coupling proves to be an effective strategy to acquire impressive attenuation of electromagnetic waves and improved overall shielding. ${ }^{45,46}$

\section{Conclusions}

In conclusion, mechanically strong RGO aerogels reinforced with one-dimensional silver nanowires were fabricated using a facile and energy-saving method. One-dimensional silver nanowires attached to RGO sheets acted as a bridge linking the sheets to facilitate formation of an electron-hopping conducting network. The addition of silver nanowires greatly enhanced the mechanical strength of the aerogel (from 1.2 to $42 \mathrm{kPa}$ ). Furthermore, excellent polarization loss and a unique nanostructure contributed to an extraordinary EMI-SE $(\sim 45.2 \mathrm{~dB})$ of the hybrid aerogel with density as low as $0.019 \mathrm{~g} \mathrm{~cm}^{-3}$, exhibiting a $264 \%$ improvement compared to the pure RGO aerogel ( $\sim 17.1 \mathrm{~dB})$. These features enable potential applications of EMIshielding materials in areas where mechanical properties are important. Accordingly, this study provides a facile approach for large-scale fabrication of high-performance electromagnetic shielding materials and broadens the applications of GO and AgNWs in multifunctional materials.

\section{Conflicts of interest}

There are no conflicts to declare.

\section{Acknowledgements}

This study was funded by the National Key Research and Development Program (2016YFB0401305). FXQ would like to thank the financial support of NSFC No. 51501162 and No. 51671171 and Fundamental Research Funds for the Central Universities No. 2018 QNA4001.

\section{Notes and references}

1 Z. H. Zeng, H. Jin, M. J. Chen, W. W. Li, L. C. Zhou and Z. Zhang, Adv. Funct. Mater., 2016, 26, 303-310.

2 Y. J. Wan, P. L. Zhu, S. H. Yu, R. Sun, C. P. Wong and W. H. Liao, Small, 2018, 14, 1800534.

3 S. Lee, I. Jo, S. Kang, B. Jang, J. Moon, J. B. Park, S. Lee, S. Rho, Y. Kim and B. H. Hong, ACS Nano, 2017, 11, 53185324.

4 W. L. Song, X. T. Guan, L. Z. Fan, W. Q. Cao, C. Y. Wang and M. S. Cao, Carbon, 2015, 93, 151-160.

5 Y. Zhang, Y. Huang, T. F. Zhang, H. C. Chang, P. S. Xiao, H. H. Chen, Z. Y. Huang and Y. S. Chen, Adv. Mater., 2015, 27, 2049-2053.
6 S. H. Lee, D. Kang and I. K. Oh, Carbon, 2017, 111, 248-257.

7 B. Shen, W. T. Zhai and W. G. Zheng, Adv. Funct. Mater., 2014, 24, 4542-4548.

8 L. Kheifets, A. Afifi and R. Shimkhada, Environ. Health Perspect., 2006, 114, 1532-1537.

9 Z. Y. Huang, H. H. Chen, Y. Huang, Z. Ge, Y. Zhou, Y. Yang, P. S. Xiao, J. J Liang, T. F. Zhang, Q. Shi, G. H. Li and Y. S. Chen, Adv. Funct. Mater., 2017, 28, 1704363.

10 Q. Song, F. Ye, X. W. Yin, W. Li, H. J Li, Y. S. Liu, K. Z. Li, K. Y. Xie, X. H. Li, Q. G. Fu, L. F. Cheng, L. T. Zhang and B. Q. Wei, Adv. Mater., 2017, 29, 1701583.

11 Y. Wu, Z. Y. Wang, X. Liu, X. Shen, Q. B. Zheng, Q. Xue and J. K. Kim, ACS Appl. Mater. Interfaces, 2017, 9, 9059-9069.

12 X. Y. Sun, X. Liu, X. Shen, Y. Wu, Z. Y. Wang and J. K. Kim, Composites, Part A, 2016, 85, 199-206.

13 T. K. Gupta, B. P. Singh, R. B. Mathur and S. R. Dhakate, Nanoscale, 2013, 6, 842-851.

14 J. B. Xi, Y. L. Li, E. Z. Zhou, Y. J. Liu, W. W. Gao, Y. Guo, J. Ying, Z. C. Chen, G. G. Chen and C. Gao, Carbon, 2018, 135, 44-51.

15 W. X. Yang, Z. D. Zhao, K. Wu, R. Huang, T. Y. Liu, H. Jiang, F. Chen and Q. Fu, J. Mater. Chem. C, 2017, 5, 3748-3756.

16 B. Shen, Y. Li, D. Yi, W. T. Zhai, X. C. Wei and W. G. Zheng, Carbon, 2016, 102, 154-160.

17 L. C. Jia, W. J. Sun, C. G. Zhou, D. X. Yan, Q. C Zhang and Z. M. Li, J. Mater. Chem. C, 2018, 6, 9166-9174.

18 S. G. Bi, L. Y. Zhang, C. Z. Mu, M. Liu and X. Hu, Appl. Surf. Sci., 2017, 412, 529-536.

19 L. Y. Zhang, M. Liu, S. G. Bi, L. P. Yang, S. Roy, X. Z. Tang, C. Z. Mu and X. Hu, J. Colloid Interface Sci., 2017, 493, 327333.

20 A. V. Menon, G. Madras and S. Bose, Chem. Eng. J., 2018, 352, 590-600.

21 D. X. Yan, P. G. Ren, H. Pang, Q. Fu, M. B. Yang and Z. M. Li, J. Mater. Chem., 2012, 22, 18772-18774.

22 C. H. Cui, D. X. Yan, H. Pang, L. C. Jia, X. Xu, S. Yang, J. Z. Xu and Z. M. Li, Chem. Eng. J., 2017, 323, 29-36.

23 F. Shahzad, P. Kumar, Y. H. Kim, S. M. Hong and C. M. Koo, ACS Appl. Mater. Interfaces, 2016, 8, 9361-9369.

24 F. Shahzad, P. Kumar, S. Yu, S. Lee, Y. H. Kim, S. M. Hong and C. M. Koo, J. Mater. Chem. C, 2015, 3, 9802-9810.

25 J. Q. Ling, W. T. Zhai, W. W. Feng, B. Shen, J. F. Zhang and W. G. Zheng, ACS Appl. Mater. Interfaces, 2013, 5, 2677-2684.

26 H. B. Zhang, Q. Yan, W. G. Zheng, Z. X. He and Z. Z. Yu, ACS Appl. Mater. Interfaces, 2011, 3, 918-924.

27 X. H. Ma, B. Shen, L. H. Zhang, Y. F. Liu, W. T. Zhai and W. G. Zheng, Compos. Sci. Technol., 2018, 158, 86-93.

28 S. Mondal, S. Ganguly, P. Das, P. Bhawal, T. K. Das, L. Nayak, D. Khastgir and N. C. Das, Cellulose, 2017, 24, 5117-5131.

29 H. D. Huang, C. Y. Liu, D. Zhou, X. Jiang, G. J. Zhong, D. X. Yan and Z. M. Li, J. Mater. Chem. A, 2015, 3, 4983-4991.

30 W. L. Song, M. S. Cao, M. M. Lu, J. Yang, H. F. Ju, Z. L. Hou, J. Liu, J. Yuan and L. Z. Fan, Nanotechnology, 2013, 24, 115708.

31 H. S. Yang, T. P. Zhang, M. Jiang, Y. X. Duan and J. M. Zhang, J. Mater. Chem. A, 2015, 3, 19268-19272. 
32 K. Krishnamoorthy, M. Veerapandian, K. Yun and S. J. Kim, Carbon, 2013, 53, 38-49.

33 M. González, J. Baselga and J. Pozuelo, J. Mater. Chem. C, 2016, 4, 8575-8582.

34 W. L. Song, M. S. Cao, M. M. Lu, S. Bi, C. Y. Wang, J. Liu, J. Yuan and L. Z. Fan, Carbon, 2014, 66, 67-76.

35 Z. P. Chen, C. Xu, C. Q. Ma, W. C. Ren and H. M. Cheng, Adv. Mater., 2013, 25, 1296-1300.

36 B. Wen, X. X. Wang, W. Q. Cao, H. L. Shi, M. M. Lu, G. Wang, H. B. Jin, W. Z. Wang, J. Yuan and M. S. Cao, Nanoscale, 2014, 6, 5754-5761.

37 M. S. Cao, W. L. Song, Z. L. Hou, B. Wen and J. Yuan, Carbon, 2010, 48, 788-796.

38 B. Shen, Y. Li, W. T. Zhai and W. G. Zheng, ACS Appl. Mater. Interfaces, 2016, 8, 8050-8057.

39 L. Kong, X. W. Yin, M. K. Han, X. Y. Yuan, Z. X. Hou, F. Ye, L. T. Zhang, L. F. Cheng, Z. W. Xu and J. F. Huang, Carbon, 2017, 111, 94-102.

40 D. X. Yan, H. Pang, B. Li, R. Vajtai, L. Xu, P. G. Ren, J. H. Wang and Z. M. Li, Adv. Funct. Mater., 2015, 25, 559566.
41 S. Singh, P. Tripathi, A. Bhatnagar, C. R. P. Patel, A. P. Singh, S. K. Dhawan, B. K. Gupta and O. N. Srivastava, RSC Adv., 2015, 5, 107083-107087.

42 Q. L. Liu, J. J. Gu, W. Zhang, Y. Miyamoto, Z. X. Chen and D. Zhang, J. Mater. Chem. C, 2012, 22, 21183-21188.

43 M. S. Cao, X. X. Wang, W. Q. Cao and J. Yuan, J. Mater. Chem. C, 2015, 3, 6589-6599.

44 F. Shahzad, M. Alhabeb, C. B. Hatter, B. Anasori, S. M. Hong, C. M. Koo and Y. Gogotsi, Science, 2016, 353, 1137-1140.

45 G. L. Wang, G. Q. Zhao, S. Wang, L. Zhang and C. B. Park, J. Mater. Chem. C, 2018, 6, 6847-6859.

46 B. Zhao, C. X. Zhao, M. Hamidinejad, C. D. Wang, R. S. Li, S. Wang, K. Yasamin and C. B. Park, J. Mater. Chem. C, 2018, 6, 10292-10300.

47 Z. Y. Min, H. Yang, F. Chen and T. R. Kuang, Mater. Lett., 2018, 230, 157-160.

48 R. Kumar, D. P. Mondal, A. Chaudhary, M. Shafeeq and S. Kumari, Composites, Part A, 2018, 112, 475-484. 